\section{P221 ADVANCE CARE PLANNING IN CHRONIC OBSTRUCTIVE PULMONARY DISEASE: A QUALITATIVE STUDY OF PATIENTS' VIEWS}

\author{
doi:10.1136/thoraxjnl-2011-201054c.221
}

${ }^{1} \mathrm{~A}$ MacPherson, ${ }^{2} \mathrm{C}$ Walshe, ${ }^{3} \mathrm{~V}$ 0'Donnell, ${ }^{3} \mathrm{~A}$ Vyas. ${ }^{1}$ North Western Deanery, Preston, UK; ${ }^{2}$ University of Manchester, Manchester, UK; ${ }^{3}$ Royal Preston Hospital, Preston, UK

Introduction and objectives There are concerns that end of life care for people with chronic obstructive pulmonary disease (COPD) is inadequate. Advance care planning is recommended for these patients, with an aim of reducing unwanted interventions and influencing place of death, but it is currently rarely done. Healthcare professionals' perspectives on the reasons for this have been sought, but there is little information about patients' views. This research explores the opinions of patients with severe COPD on advance care planning, and how they feel it could be integrated into their care.

Methods A qualitative interview strategy was adopted, drawing from grounded theory techniques. Patients with severe COPD (according to Gold Standards Framework criteria ${ }^{1}$ ) were recruited from two primary and secondary care settings ( $n=10,9 M, 1 F)$. Interviews using a broad topic guide were conducted in participants' homes, audio recorded and transcribed verbatim. Analysis was inductive, generating themes using techniques of constant comparison.

Results People with severe COPD wanted more information about diagnosis, and more discussion of their prognosis and future. They very much wanted to be involved in decisions about their current care. They were happy to discuss general treatment preferences for the future, but not to make binding advance decisions. They expected the initiation of these discussions to come from the healthcare team, especially those they feel they know. When information was inadequate or they felt they had not been included in decisions, the relationship with their healthcare team was damaged. Discussing these issues does not cause undue distress.

Conclusions A focus on encouraging formal binding advance decisions for people with severe COPD is not helpful. An advance care planning approach involving repeated discussions of disease status, prognosis and general preferences for future care would be more effective. Healthcare professionals should take responsibility for initiating these discussions. As well as improving end of life care, this improved information provision could improve relationships between patients and healthcare professionals, as well as enabling patients to be more involved in current decisions about their care.

\section{REFERENCE}

1. The Gold Standards Framework "Prognostic Indicator Guidance". 2008;Revised version 5:2

\section{P222 MULTI-DISCIPLINARY TEAM ATTITUDES TOWARDS END- OF-LIFE CARE IN SEVERE COPD}

doi:10.1136/thoraxjnl-2011-201054c.222

B Turnpenny, K Shepherd, R Barraclough, C Haskins. University Hospital of South Manchester, Manchester, Lancashire, UK

Introduction Chronic obstructive pulmonary disease (COPD) is the fourth leading cause of death globally. The physical and psychological symptom burden in severe COPD is significant and yet many of these symptoms go untreated (Elkington et al, 2005). Patients with COPD have far less specialist palliative care input than patients with lung cancer despite a symptom burden at the end of life similar, or indeed greater, than patients with lung cancer (Gore et al, 2000).

Methods An anonymous questionnaire was completed by respiratory healthcare professionals including respiratory doctors of all grades, physiotherapists and nurses of different backgrounds. The questions focused on perceived ease of predicting prognosis in severe COPD, the initiating of end-of-life discussions in this group, and training received in palliative care.

Results 100 questionnaires were completed. 67 participants were doctors, from house officers to consultants, 5 physiotherapists, 9 respiratory specialist nurses, 8 Macmillan nurses and 12 respiratory ward nurses. The questionnaire found $76 \%$ of participants felt it was a very important or important part of their role to initiate endof-life discussions in severe COPD and $2 \%$ felt it was not, or definitely not part of their role. Participants initiated such discussions frequently or very frequently in $20 \%$, and infrequently or very infrequently in $55 \%$. Prognosis in severe COPD was difficult or very difficult in $63 \%$ and straightforward or very straightforward in $6 \%$. This is consistent with literature (Gott et al, 2009). Training participants had received in palliating symptoms in this patient group was significant or extensive in $25 \%$ and minimal or little in $41 \%$. Training in initiating end-of-life discussions was significant or extensive in 19\% and minimal or little in 44\%. Training in services available to this group of patients was significant or extensive in $17 \%$ and minimal or little in $42 \%$.

Conclusion The need to initiate end-of-life discussions in severe COPD is felt to be important to the multidisciplinary team's role in palliating such patients, yet very few regularly have such discussions. A lack of training and self-reported difficulty in predicting prognosis in this group are perceived to be great, and are suggested as possible reasons for this imbalance.

\section{P223 END OF LIFE DISCUSSION IN PULMONARY REHABILITATION: TOO SCARY OR WORTH A TRY?}

doi:10.1136/thoraxjnl-2011-201054c.223

${ }^{1} \mathrm{~S}$ Robinson, 'L Wilson, 'M Oayoom, 'S Dawson, 'L Killeen, ${ }^{1} \mathrm{~T}$ Shturova, ${ }^{2} \mathrm{~S}$ Patel. ${ }^{1}$ Central London Community Healthcare, London, UK; ${ }^{2}$ Imperial College Healthcare NHS Trust, London, UK

Background End of life is a topic which may be included in pulmonary rehabilitation (PR) education sessions. Patient expectations from such a session, its optimal content, and who should deliver it is unknown.

Method The community PR programme in Hammersmith and Fulham has included a session entitled "Managing the Final Stages of COPD" since April 2010. Apprehensions and training needs around this subject were identified in respiratory staff running PR and therefore a community matron with an interest in palliative and end of life care led the sessions. Twelve-sixty min end of life discussion sessions were delivered from April 2010 to June 2011. The content was based around the eponymous British Lung Foundation (BLF) leaflet. Discussions focused principally on making a will, arranging a funeral and considering a Power of Attorney or Advance Decision. Participants were given advance notice and an opportunity to "opt out".

Results 72 patients attended these sessions (average number 6). None opted out. One patient asked for more information afterwards. Two partcipants became upset during the session; one opted to attend in spite of a recent bereavement and the other reported "feeling relieved". Evaluation responses are shown in Abstract P223 table 1.

Conclusion Advanced care planning is well received and can be delivered as part of community PR education. Respiratory staff leading PR are now confident about delivering this themselves. However this setting is not able to address the advanced care planning needs of specific patients with adverse prognostic indicators and this needs separate planning. 